\author{
Jasper D. Sluimer \\ Wiesje M. van der Flier \\ Giorgos B. Karas \\ Ronald van Schijndel \\ Josephine Barnes \\ Richard G. Boyes \\ Keith S. Cover \\ Sílvia D. Olabarriaga \\ Nick C. Fox \\ Philip Scheltens \\ Hugo Vrenken \\ Frederik Barkhof
}

\section{Accelerating regional atrophy rates in the progression from normal aging to Alzheimer's disease}

Received: 23 January 2009

Revised: 15 April 2009

Accepted: 22 May 2009

Published online: 18 July 2009

(C) The Author(s) 2009.

This article is published with open access at Springerlink.com

J. D. Sluimer - G. B. Karas · F. Barkhof Department of Diagnostic Radiology, VU University Medical Centre, Amsterdam, The Netherlands

J. D. Sluimer - W. M. van der Flier .

G. B. Karas · P. Scheltens ·

H. Vrenken · F. Barkhof

Alzheimer Centre,

VU University Medical Centre,

Amsterdam, The Netherlands

J. D. Sluimer - G. B. Karas •

R. van Schijndel · H. Vrenken ·

F. Barkhof

Image Analysis Centre,

VU University Medical Centre,

Amsterdam, The Netherlands

W. M. van der Flier - N. C. Fox •

P. Scheltens

Department of Neurology,

VU University Medical Centre,

Amsterdam, The Netherlands

K. S. Cover · H. Vrenken

Department of Physics and Medical

Technology, VU University

Medical Centre,

Amsterdam, The Netherlands
R. van Schijndel

Department of Informatics,

VU University Medical Centre,

Amsterdam, The Netherlands

J. Barnes · R. G. Boyes · N. C. Fox

Dementia Research Centre,

UCL, Institute of Neurology,

London, UK

S. D. Olabarriaga

Department of Clinical Epidemiology,

Biostatistics and Bioinformatics,

Academic Medical Centre,

University of Amsterdam,

Amsterdam, The Netherlands

J. D. Sluimer $(\bowtie)$

Department of Diagnostic Radiology

and Alzheimer Centre,

VU University Medical Centre,

PO Box 7057, 1007 MB

Amsterdam, The Netherlands

e-mail: jd.sluimer@vumc.nl

Tel.: +31-20-4441079

Fax: +31-20-4440715

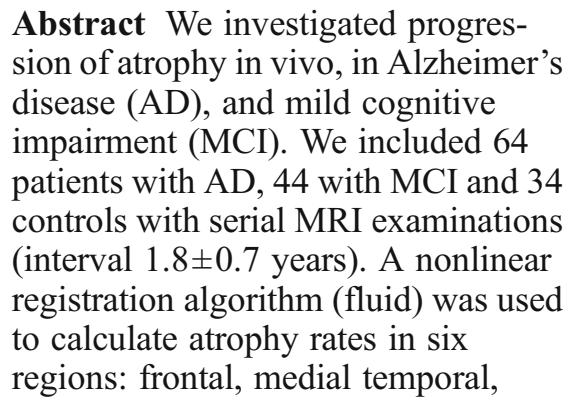

temporal (extramedial), parietal, occipital lobes and insular cortex. In MCI, the highest atrophy rate was observed in the medial temporal lobe, comparable with $\mathrm{AD}$. AD patients showed even higher atrophy rates in the extramedial temporal lobe. Additionally, atrophy rates in frontal, parietal and occipital lobes were increased. Cox proportional hazard models showed that all regional atrophy rates predicted conversion to $\mathrm{AD}$. Hazard ratios varied between 2.6 $(95 \%$ confidence interval $(\mathrm{CI})=1.1-$ 6.2) for occipital atrophy and 15.8 (95\% CI $=3.5-71.8)$ for medial temporal lobe atrophy. In conclusion, atrophy spreads through the brain with development of AD. MCI is marked by temporal lobe atrophy. In AD, atrophy rate in the extramedial temporal lobe was even higher. Moreover, atrophy rates also accelerated in parietal, frontal, insular and occipital lobes. Finally, in nondemented elderly, medial temporal lobe atrophy was most predictive of progression to $\mathrm{AD}$, demonstrating the involvement of this region in the development of $\mathrm{AD}$.

Keywords Alzheimer's disease . Magnetic resonance imaging . Atrophy - Image processing . Computer-assisted 


\section{Introduction}

Alzheimer's disease (AD) is a neurodegenerative disease, characterised by progressive cognitive decline and cerebral atrophy. Patients with mild cognitive impairment (MCI) have measurable cognitive deficits, but do not fulfil criteria for dementia [1]. Subjects with MCI are at an increased risk of developing $\mathrm{AD}$ with about $10-15 \%$ progressing to $\mathrm{AD}$ per year: in these subjects MCI may be considered to be a transitional phase for $\mathrm{AD}$ [2].

Neuropathological studies suggest that Alzheimer's pathology spreads throughout the brain in a relatively predictable fashion and starts well before clinical onset of disease [3]. However, by definition, neuropathological studies are post hoc and cross-sectional in design and clearly cannot track disease progression in the individual. Magnetic resonance imaging (MRI) of the brain shows atrophy in vivo, particularly involving the medial temporal lobe, early in the disease [4]. In contrast to neuropathological studies, serial MRI is feasible and enables in vivo study of progression of the disease throughout the brain [4, 5]. Serial measures allow each subject to act as their own control and thereby avoid the wide variability in brain morphology between subjects. In particular hippocampal atrophy rates have shown to be sensitive markers of $\mathrm{AD}$, and predict progression of cognitive decline more accurately than cross-sectional volumes [6-8]. However, whole-brain atrophy rates also distinguish patients with $\mathrm{AD}$ and $\mathrm{MCI}$ from controls, suggesting the additional early involvement of brain regions other than the hippocampus $[5,9,10]$.

We studied progression of atrophy in six lobar brain regions in $\mathrm{AD}$, MCI and control subjects. We hypothesised that atrophy rates of MCI patients would be highest in the medial temporal lobes, consistent both with this region's early pathological involvement and its association with memory function - often the presenting symptom [3]. With advancing disease, we expected atrophy rates of $\mathrm{AD}$ patients to increase in other brain regions as well. This study aimed to compare the pattern of regional atrophy rates in $\mathrm{AD}, \mathrm{MCI}$ and controls. In addition, we investigated associations between regional atrophy rates and clinical progression to $\mathrm{AD}$ in initially nondemented patients.

\section{Material and methods}

\section{Patients}

\section{Baseline clinical assessment}

We included 64 patients with probable AD, 44 patients with MCI and 34 controls. All patients underwent a standardised clinical assessment including medical history, physical and neurological examination and psycho- metric evaluation. All subjects had serial volumetric MR imaging. The mini-mental state examination (MMSE) was used as a measure of general cognitive function [11]. Diagnoses were established during a multidisciplinary consensus meeting according to the Petersen criteria for MCI [12] and the NINCDS-ADRDA (National Institute of Neurological and Communicative Diseases and Stroke/ Alzheimer's Disease and Related Disorders Association) criteria for probable $\mathrm{AD}$ [13]. The control group consisted of 26 patients who presented at our memory clinic with subjective complaints, but who had normal clinical investigations and did not have significant cognitive deficits (i.e. MCI criteria were not fulfilled). Additionally, we included eight volunteers without cognitive complaints, who were willing to undergo the same diagnostic procedure as patients attending our memory clinic. The study was approved by the institutional ethical review board. All participants (or caregivers) gave written informed consent.

\section{Clinical assessment at follow-up}

Diagnostic classification of nondemented participants (MCI and controls) was re-evaluated at follow-up. The clinical diagnosis of dementia was determined according to published consensus criteria [12-16]. Within the MCI group, 16 patients had remained stable, 23 progressed to $\mathrm{AD}$, two to frontal-temporal lobar degeneration (FTLD) [14], two to vascular dementia (VaD) [15] and one to dementia with Lewy bodies (DLB) [16]. Within the group of controls, three progressed to MCI, three to AD and one to FTLD, whilst 27 controls remained stable.

\section{MRI}

MR imaging was performed on a 1.0-T Siemens Magnetom Impact Expert (Siemens AG, Erlangen, Germany) and included coronal T1-weighted 3D MPRAGE volumes (magnetization prepared rapid acquisition gradient echo; single slab, 168 slices; matrix size $256 \times 256$; FOV $250 \mathrm{~mm}$; voxel size $1 \times 1 \times 1.5 \mathrm{~mm}$; TR= $15 \mathrm{~ms} ; \mathrm{TE}=7 \mathrm{~ms}$; TI $=300 \mathrm{~ms}$; flip angle $15^{\circ}$ ). For inclusion subjects had to have had two MR examinations without artefacts, performed on the same MR system using the same imaging protocol. Images were reviewed by a neuroradiologist to exclude non-neurodegenerative pathology that could explain the cognitive impairment. Patients with baseline MR examinations that fulfilled radiological criteria of the NINDS-AIREN for vascular dementia were excluded [15].

Regional atrophy rates between two time-points were measured from the 3D-T1 MPRAGE images using fluid registration, a nonlinear matching algorithm $[17,18]$. This algorithm requires the baseline and repeat image to 
be aligned, and intensity to be normalised within and between volumes. We used the following preprocessing steps:

- All images were bias-corrected using N3 software (http://www.bic.mni.mcgill.ca/software/N3/) [19].

- Images from the two time-points of each subject were linearly coregistered to a halfway position, using the skull-based scaling constraint as implemented in the SIENA software (structural brain change analysis, for estimating brain atrophy), part of the FSL suite (http:// www.fmrib.ox.ac.uk/fsi/) [20].

- Residual bias field differences between the two timepoints were then removed using differential bias collection (DBC) as described by Lewis and Fox [21].

- A brain mask was extracted from the resulting images using BET (brain extraction tool) combined with registration of a standard mask using FLIRT (FMRIB's linear image registration tool) [22].

- The brain mask was expanded to induce a Gaussian signal drop-off in space around the brain tissue.

After preprocessing, nonlinear registration between the registered baseline and repeat images was performed, using a nonlinear voxel-compression technique (fluid) [17]. This technique uses a viscous fluid model to compute the transformation required to bring one image in register with the other, using cross correlation as a similarity function. The data analysis for the whole data set required around 1,200 computing hours, and was performed in the GRID infrastructure provided by the Virtual Laboratory for eSciences project (www.vl-e.nl). By using trivially parallel computing the entire dataset could be processed in a single weekend. Visual inspection of the results confirmed that in all cases the registration had succeeded (i.e. all image pairs were well matched and the algorithm had succeeded in deforming the latter MR examinations compared with the baseline-effectively removing the effects of atrophy). From the transformation field created by each registration, we generated a map of Jacobian determinants; these are a measure of the volume change (compression or expansion) at each voxel required to match the examinations precisely. Examples of these voxel-compression (Jacobian) maps are shown in Fig. 1.

We then defined six lobar regions as follows: first brain tissue was separated from nonbrain using FAST (FMRIB's automated segmentation tool) [22]; secondly, the AAL mask (automated anatomical labeling)[23] was coregistered to the individual data. We used the subdivisions of the AAL mask of six predefined lobar regions: frontal, medial temporal (hippocampus, parahippocampal gyrus, and amygdala), temporal (excluding medial temporal), parietal, occipital lobes and insular cortex. For every patient, we averaged the relative volume change over all voxels in each region, and divided by the follow-up time in years between MR examinations to obtain annualized regional atrophy rates.

\section{Statistical analysis}

Statistical analysis was performed with SPSS 12.0 (2003, Chicago, Illinois). Follow-up time was defined as the interval in years between baseline and repeat MR examinations. Diagnostic groups were compared with chi-squared tests for sex. For continuous variables we used analysis of variance (ANOVA), with age and sex as covariates. Post hoc comparisons were performed using Bonferroni tests. Differences in regional atrophy rates between groups were assessed using ANOVA for repeated measures with region as within-subjects factor, diagnosis as between-subjects factor, and age and sex as covariates. In addition, within the group of initially nondemented participants we assessed the predictive value of regional atrophy rates, by using Cox proportional hazards models, which account for variability in length of follow-up. Results are presented as hazard ratios (HRs) with 95\% confidence interval $(\mathrm{CI})$. The main outcome was progression to dementia, while the secondary outcome was progression to $A D$, which excludes six cases who developed a different type of dementia. Regional atrophy rates (dichotomized into high and low rates of atrophy, based on the median) were used as independent variables in separate models, adjusted for age and sex. Time-to-event curves were constructed with the Kaplan-Meier method. Statistical significance was set at $p<0.05$.

\section{Results}

Demographics and clinical data are presented by patient group in Table 1. No between-group difference in sex or follow-up time was found. MCI patients were older than AD patients and controls. Frontal, medial temporal, temporal (excluding medial temporal), parietal, occipital and insular atrophy rates are given in Table 1. ANOVA for repeated measures with region as within-subjects factor, group as between-subjects factor, and age and sex as covariates showed main effects of diagnostic group ( $p<$ $0.001)$ and region $(p<0.001)$, and an interaction between diagnosis and region $(p<0.001)$, indicating different patterns of regional atrophy rates according to diagnostic group. Figure 2 shows that for MCI patients, the highest annualized atrophy rates were observed not only in the medial temporal lobe, but also in the remaining (extramedial) part of the temporal lobe. With progression of the disease, the atrophy appears to spread more widely through the brain, with patients with clinically established $\mathrm{AD}$ showing higher atrophy rates in temporal, frontal, parietal and occipital lobes. The atrophy rate in the medial temporal lobe appears to already have reached its maximum at a stage prior to clinical diagnosis, as this rate did not increase further in patients with $\mathrm{AD}$ when compared with MCI patients.

Subsequently, we assessed the predictive value of regional atrophy rates for the prediction of dementia in 
Fig. 1 Baseline MR examination (left), repeat MR examination (middle) and colour overlay overlaid on the baseline examination (right) of four individual patients: a 50-year-old control subject, who presented at the memory clinic with subjective memory complaints $(A)$; a $72-$ year-old MCI patient who remained stable during follow-up $(B)$; a 69-year-old MCI patient who progressed to $\mathrm{AD}$ during follow-up $(C)$; a 64-year-old, moderately demented AD patient $(D)$. Baseline and repeat examinations were affine-registered. The result of the nonlinear registration is presented as a colour overlay applied to the baseline examination (representing the local Jacobian of the calculated deformation field), in order to highlight regions of structural expansion and contraction. Green and blue represent moderate to severe contraction (atrophy), yellow and red moderate to severe expansion. The overlay image was masked with a dilated mask to also show expansion of peripheral CSF spaces

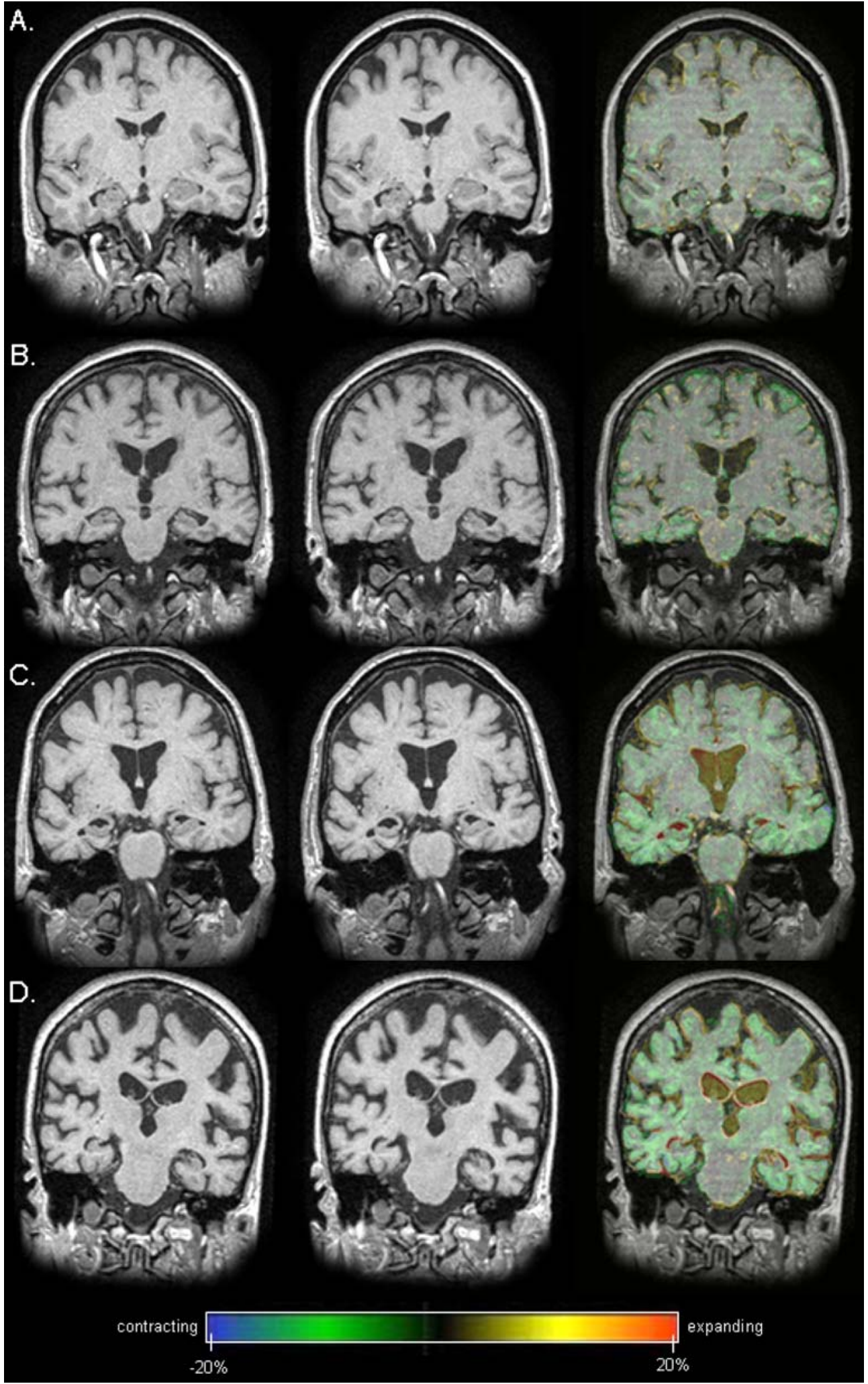


Table 1 Demographic and clinical variables by diagnostic group

\begin{tabular}{|c|c|c|c|c|}
\hline & Controls & $\mathrm{MCI}$ & $\mathrm{AD}$ & Overall $p$ value \\
\hline Number of participants & 34 & 44 & 64 & \\
\hline \multicolumn{5}{|l|}{ Outcome (numbers) } \\
\hline Stable & 30 & 16 & - & \\
\hline Remaining stable & $3 \mathrm{AD}$ & $23 \mathrm{AD}$ & & \\
\hline Converting & 1 other & 5 other & & \\
\hline Sex (female/male) & $16 / 18$ & $21 / 23$ & $38 / 26$ & $p=0.37$ \\
\hline Age (years) & $67(9)^{* *}$ & $71(6)$ & $67(8)^{* *}$ & $p<0.001$ \\
\hline MMSE baseline & $28(2)$ & $26(3)^{*}$ & $22(5)^{* * *, * * * *}$ & $p<0.001$ \\
\hline MR examination interval (years) & $1.9(0.9)$ & $1.9(0.7)$ & $1.7(0.6)$ & $p=0.39$ \\
\hline \multicolumn{5}{|l|}{ Regional atrophy rates (\%/year) } \\
\hline Frontal & $-0.6(0.7)$ & $-0.9(0.7)$ & $-1.3(0.8)^{* * *}$ & $p<0.001$ \\
\hline Medial temporal & $-0.6(0.7)$ & $-1.5(0.7)^{* * *}$ & $-1.5(0.7)^{* * *}$ & $p<0.001$ \\
\hline Temporal (extramedial) & $-0.6(0.5)$ & $-1.4(0.8)^{* * *}$ & $-2.2(1.0)^{* * *, * * * * *}$ & $p<0.001$ \\
\hline Parietal & $-0.5(0.5)$ & $-0.9(0.7)^{*}$ & $-1.7(0.9)^{* * *, * * * *}$ & $p<0.001$ \\
\hline Occipital & $-0.4(0.4)$ & $-0.8(0.6)^{* * *}$ & $-1.4(1.0)^{* * *, * * * *}$ & $p<0.001$ \\
\hline Insular cortex & $-0.3(0.7)$ & $-0.7(0.6)$ & $-0.8(0.8)^{* * *}$ & $p<0.001$ \\
\hline
\end{tabular}

Data are displayed as mean with standard deviation (sd) in parenthesis. Differences between groups were assessed using ANOVA (age and sex as covariates where appropriate) with post hoc Bonferroni tests. Group difference for sex was calculated with the Pearson chi-square test. Medial temporal lobe is defined as hippocampus, parahippocampal gyrus, and amygdala. Temporal lobe is defined as temporal lobe excluding medial temporal lobe

$M C I$ mild cognitive impairment, $A D$ Alzheimer's disease, MMSE mini-mental state examination ${ }^{*} p<0.05$ compared with controls; ${ }^{* *} p<0.05$ compared with MCI; ${ }^{* * *} p<0.001$ compared with controls; ${ }^{* * * *} p<0.001$ compared with MCI

initially nondemented patients (controls and MCI, $n=78$ ). Thirty-two patients progressed to dementia, while 46 patients remained stable. No differences were found between patients progressing to dementia and patients who remained stable during follow-up, for sex (female/ male $17 / 15$ versus $20 / 26 ; p=0.57$ ), age (mean \pm sd $71 \pm 6$ versus $68 \pm 9 ; p=0.14)$ or duration of follow-up (1.8 \pm 0.7 years versus $1.9 \pm 0.8$ years; $p=0.53$ ). There was a difference in baseline MMSE score between progressing and stable patients $(26 \pm 3$ versus $28 \pm 2 ; p<0.001)$. Ageand sex-adjusted Cox proportional hazard models showed that atrophy rates in all regions were associated with an increased risk of progression to dementia (Table 2). Risk estimates varied between 2.0 (95\% confidence interval, $\mathrm{CI}=0.9-4.4)$ for occipital lobe atrophy rate and $6.4(95 \%$ $\mathrm{CI}=2.4-17.3$ ) for medial temporal lobe atrophy rate. As an example, Fig. 3 shows the Kaplan-Meier survival curve for the medial temporal lobe. When the analysis was repeated and restricted to progression to AD (excluding six patients who developed other types of dementia) the risk estimates were even higher. Atrophy rates in the medial temporal lobe remained the best predictor in the model, HR 15.8 (95\% CI $=3.5-71.8)$. In addition, the temporal lobe (nonmedial) and parietal lobe were good predictors of progression to $\mathrm{AD}$. When we repeated the analysis with additional adjustment for baseline MMSE, results remained largely unaltered (data not shown).

\section{Discussion}

The main finding of our study is that the pattern of regional atrophy rates differs in patients with MCI and AD when compared with controls. These data suggest how atrophy rates accelerate through the brain with the progression of cognitive decline. In MCI, the temporal lobe shows the greatest atrophy rate, but atrophy already extends beyond the medial temporal lobe, with atrophy rates in the extramedial part of the temporal lobe even at this stage appearing equally high. Atrophy rates in the medial temporal lobe were no higher in the AD patients than in the MCI patients, implying that rate of neurodegeneration in this region is already maximal prior to clinical diagnosis. The progression to $\mathrm{AD}$ is characterised by increasing atrophy rates in the rest of the temporal lobe, and atrophy also accelerating in parietal, frontal and occipital lobes. Nonetheless, medial temporal lobe atrophy was most predictive of progression to dementia in initially nondemented patients. Mainly subjects with a higher than median atrophy rate were associated with a 15 -fold higher risk of developing $\mathrm{AD}$, when compared with those with a lower than median atrophy rate during follow-up.

Previous studies report increased cerebral atrophy rates and ventricular enlargement in $\mathrm{AD}$ versus controls [9, 24]. Most cross-sectional MRI studies have focussed on atrophy of medial temporal lobe structures, commonly 


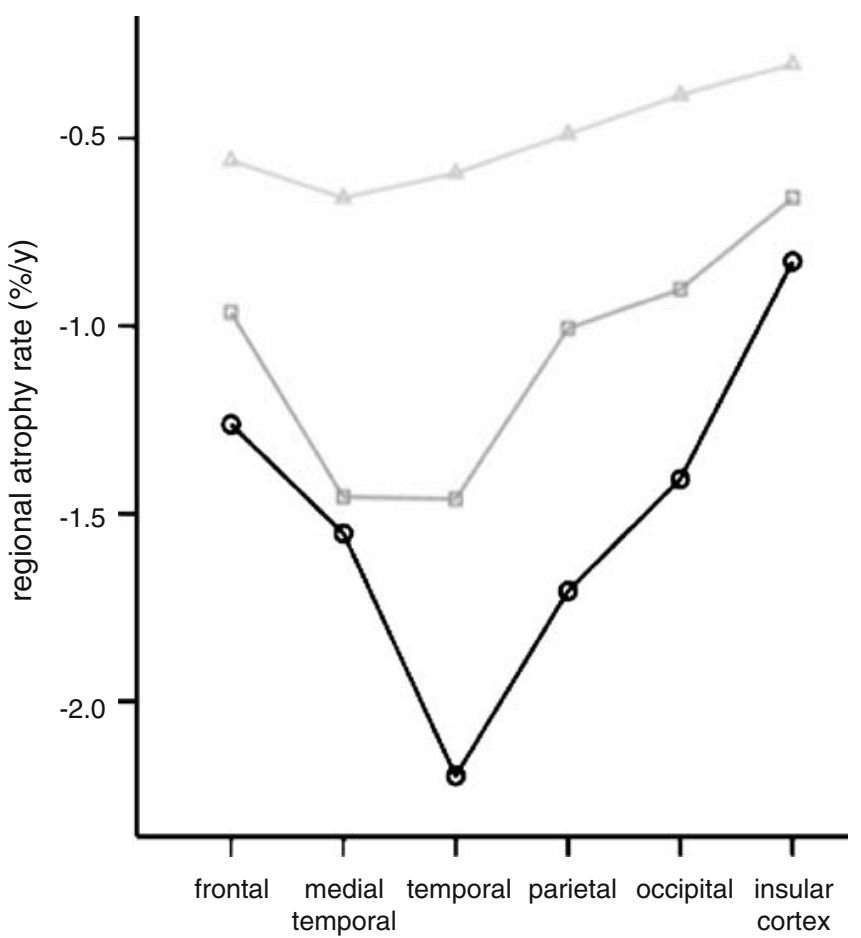

Fig. 2 Regional atrophy rate in six predefined lobar regions are presented by diagnostic group: frontal, medial temporal (hippocampus, amygdala, parahippocampal gyrus), temporal (extramedial), parietal, occipital and insular lobe. In controls, atrophy rates are around $0.5 \%$ year for each region. In MCI patients, atrophy rates start to accelerate mainly in the medial temporal, and remaining temporal lobe (extramedial), and to a lesser extent in the other regions. $\mathrm{AD}$ is characterised by a further increase in atrophy rate in the remainder of the temporal lobe, parietal, frontal, occipital and insular lobe. Medial temporal lobe atrophy rates appear to be at a maximum, in the preclinical stage, since the rate is comparable to that of MCI patients. $\Delta=$ controls (light grey line); $\square=$ MCI (dark grey line); $\mathrm{O}=\mathrm{AD}$ (black line)

using volumetric measures. These studies have shown that hippocampal volumes at the time of clinical diagnosis are on average $18 \%$ lower when compared with controls [6, 25]. Whole-brain volumes are decreased in AD also, and tend to be around 5\% lower-this volume loss cannot be

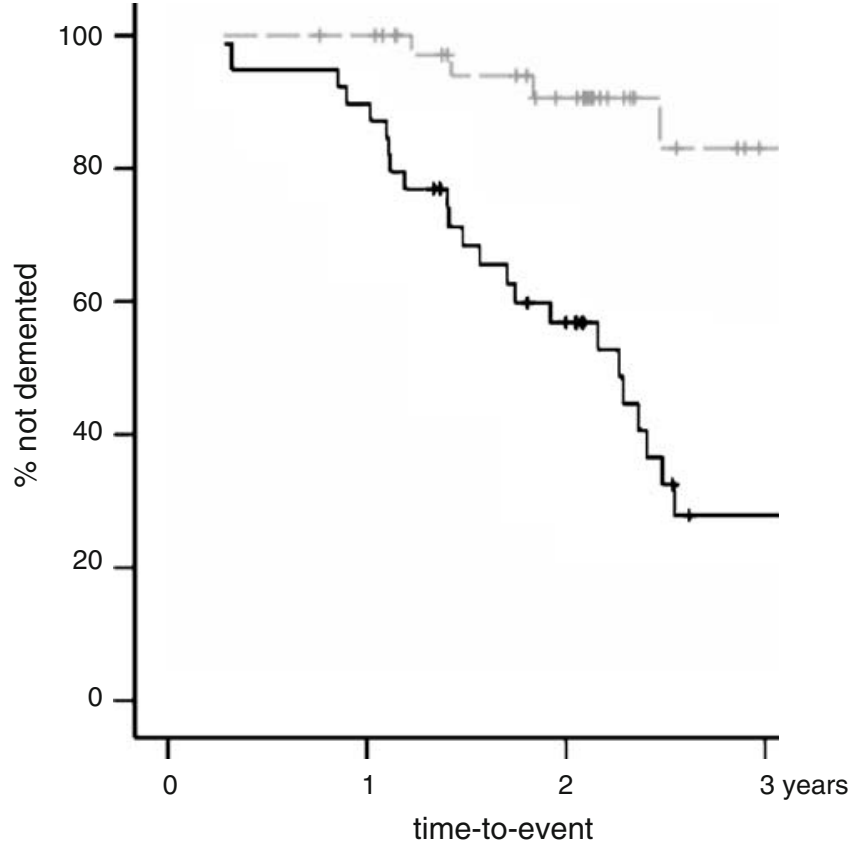

Numbers at risk $(\mathrm{n}=78)$ :

Low atrophy rate $(\mathrm{n}=39)$

High atrophy rate $(n=39)$

25

7

19

Fig. 3 Kaplan-Meier curve of time-to-conversion in initially nondemented patients $(n=78)$ dependent on medial temporal lobe atrophy rate. Nondemented patients were dichotomised into either the high or the low category, based on median medial temporal lobe atrophy rate $(-0.9 \% /$ year $)$. Numbers at risk are displayed below graph. Participants reaching end of follow-up period without progression to dementia were censored. Low atrophy rate (dashed grey line); high atrophy rate of the medial temporal lobe (solid black line); $+=$ censored

accounted for by medial temporal lobe damage and implies widespread atrophy beyond this region [10]. Voxel-based morphometry studies have shown using cross-sectional comparisons that in addition to atrophy of medial temporal lobe structures, temporal-parietal atrophy is prominent in $\mathrm{AD}$ [26-28]. In MCI patients progressing to $\mathrm{AD}$ versus stable MCI patients atrophy of the temporal lobe, and to a

Table 2 Risk of progression to dementia associated with regional atrophy measures

\begin{tabular}{llll}
\hline & Median atrophy rate & $\begin{array}{l}\text { Outcome }=\text { dementia } \\
(n=78) \\
\text { \%/year (IQ range) }\end{array}$ & $\begin{array}{l}\text { Outcome }=\mathrm{AD} \\
(n=72) \\
\text { HR }(\mathrm{CI})\end{array}$ \\
\hline Frontal lobe & $-0.8(-1.2$ to -0.4$)$ & $2.2(1.0-4.9)$ & $2.8(1.1-6.8)$ \\
Medial temporal lobe & $-0.9(-1.5$ to -0.3$)$ & $6.4(2.4-17.3)$ & $15.8(3.5-71.8)$ \\
Temporal lobe & $-0.9(-1.4$ to -0.4$)$ & $3.9(1.7-9.2)$ & $6.3(2.2-18.7)$ \\
Parietal lobe & $-0.6(-1.0$ to -0.2$)$ & $3.4(1.4-8.3)$ & $5.1(1.8-14.8)$ \\
Occipital lobe & $-0.5(-0.9$ to -0.2$)$ & $2.0(0.9-4.4)$ & $2.6(1.1-6.2)$ \\
Insular cortex & $-0.4(-0.9$ to 0$)$ & $2.5(1.1-5.9)$ & $2.9(1.2-7.3)$ \\
\hline
\end{tabular}

Median atrophy rate is displayed as median (interquartile (IQ) range). Other data are presented as age- and sex-adjusted hazard ratios (HR) and $95 \%$ confidence interval (CI). In the third column, six patients who progressed to another type of dementia were excluded 
lesser extent of the parietal and frontal lobe was shown [29-31]. Longitudinal measures of atrophy, which allow changes within the individual to be assessed, have been shown to be more sensitive at detecting group differences than cross-sectional volumes, in part because they are less susceptible to intersubject variability $[4,32,33]$. A number of studies described the pattern of longitudinal atrophy in relatively small groups of sporadic and familial $\mathrm{AD}$ patients. They found increased atrophy in the medial temporal lobe over time, but also more widespread throughout the brain, in the parietal and frontal lobe [17, 34]. Longitudinal studies report that hippocampal atrophy rates increase in MCI [6-8]. We extend these findings, by providing rates of atrophy in six brain regions. Our study confirms that the medial temporal lobe is affected at an early stage of the disease. Additionally, we found the mean atrophy rate in the remainder of the temporal lobe to be increased at an early stage as well, before a clinical diagnosis could be made with certainty. Regional atrophy rates in the remainder of the cortex further accelerated in AD.

Among the limitations of our study is the fact that the control group included individuals with subjective complaints. At baseline, we found no differences between healthy volunteers and patients with subjective complaints, and we therefore pooled these subjects in one control group. However, patients presenting at a memory clinic with subjective complaints are known to have a higher risk of developing $\mathrm{AD}[35,36]$. In fact, three patients converted to $\mathrm{AD}$ and one to FTLD. One could argue that these patients should have been excluded, as they did not remain control-like throughout the study. However, we feel that, since they fulfilled initial inclusion criteria, excluding these patients would bias the results. Looking back at the regional atrophy rates, the patients who progressed to $\mathrm{AD}$ showed high atrophy rates, congruent with their clinical progression. Regional (including frontal) atrophy rates of the patient who progressed to FTLD were not remarkable. A second limitation was that no postmortem verification of diagnosis, which is considered the gold standard for diagnosing $\mathrm{AD}$, was available. We therefore cannot exclude the possibility that some of our patients were misdiagnosed. Even though our MCI patients fulfilled the Petersen criteria, generally regarded as predictive of $\mathrm{AD}$, five patients progressed to another type of dementia. When we visually inspected the individual data, the FTLD progressors showed relatively high frontal atrophy rates, while the pattern was more generalized for the two patients with $\mathrm{VaD}$ and the patient who progressed to DLB. Remarkably, none of these five patients showed predominant medial temporal lobe atrophy, as was generally observed in $\mathrm{MCI}$ patients progressing to $\mathrm{AD}$.

Among the strengths of our study was the relatively large sample, in which we studied regional atrophy rates, using a robust method. We used fluid registration, a nonlinear registration algorithm to match two MR examinations of each individual. This fully automated voxel-compression technique has the advantage that changes at a lobar level can be assessed without time-consuming manual measurement of regional volumes. Furthermore, the nonlinear registration matches examinations with shape change as well as volume loss. This means that the structural readjustments, that are an inevitable consequence of neurodegeneration, do not confound assessment of the distribution of loss.

The results of our study are in agreement with what has been published about the progression of atrophy through the brain in neuropathological studies regarding $\mathrm{AD}[3$, 37-40]. Neuropathological studies report that AD pathology spreads through the brain in a relatively predictable fashion [3]. For neurofibrillary tangles the progression can be separated into six stages: stages I and II show alterations in the transentorhinal regions, stages III and IV are known as the limbic stage, while stage V and VI are marked by widespread isocortical involvement. The accumulation of amyloid deposits can be divided into three stages: stage A shows initial deposits in the basal portions of the isocortex, stage B shows amyloid in virtually all isocortical association areas, but the hippocampal formation is only mildly involved, while in stage $\mathrm{C}$ end-stage deposits can be observed throughout the isocortex. This study demonstrated that the pattern of atrophy, observed in vivo using serial MRI, more closely resembles the pattern of accumulating neurofibrillary tangles. Furthermore, the early involvement of the medial temporal lobe, as observed in neuropathological studies, has shown to be of clinical importance in the detection of incipient $\mathrm{AD}$ in this study.

Open Access This article is distributed under the terms of the Creative Commons Attribution Noncommercial License which permits any noncommercial use, distribution, and reproduction in any medium, provided the original author(s) and source are credited.

\section{Reference}

1. Petersen RC, Doody R, Kurz A et al (2001) Current concepts in mild cognitive impairment. Arch Neurol 58:1985-92

2. Gauthier S, Reisberg B, Zaudig M et al (2006) Mild cognitive impairment. Lancet 367:1262-70
3. Braak H, Braak E (1991) Neuropathological stageing of Alzheimer-related changes. Acta Neuropathol (Berl) $82: 239-59$

4. Fox NC, Scahill RI, Crum WR et al (1999) Correlation between rates of brain atrophy and cognitive decline in AD. Neurology 52:1687-9
5. Jack CR, Shiung MM, Weigand SD et al (2005) Brain atrophy rates predict subsequent clinical conversion in normal elderly and amnestic MCI. Neurology 65:1227-1231 
6. Jack CR Jr., Petersen RC, Xu Y et al (2000) Rates of hippocampal atrophy correlate with change in clinical status in aging and AD. Neurology 55:484-89

7. Tapiola T, Pennanen C, Tapiola $\mathrm{M}$ et al (2006) MRI of hippocampus and entorhinal cortex in mild cognitive impairment: a follow-up study. Neurobiol Aging 29:31-38

8. van de Pol LA, van Der Flier WM, Korf ES et al (2007) Baseline predictors of rates of hippocampal atrophy in mild cognitive impairment. Neurology 69:1491-7

9. Jack CR, Shiung MM, Gunter JL et al (2004) Comparison of different MRI brain atrophy, rate measures with clinical disease progression in AD. Neurology 62:591-600

10. Sluimer JD, van Der Flier WM, Karas GB et al (2007) Whole-brain atrophy rate and cognitive decline: a longitudinal MRI study of memory clinic patients. Radiology 248:590-598

11. Folstein MF, Folstein SE, Mchugh PR (1975) "Mini-mental state". A practical method for grading the cognitive state of patients for the clinician. J Psychiatr Res 12:189-98

12. Petersen RC, Stevens JC, Ganguli M et al (2001) Practice parameter: early detection of dementia: mild cognitive impairment (an evidence-based review). Report of the Quality Standards Subcommittee of the American Academy of Neurology. Neurology 56:1133-42

13. McKhann G, Drachman D, Folstein M et al (1984) Clinical diagnosis of Alzheimer's disease: report of the NINCDS-ADRDA Work Group under the auspices of Department of Health and Human Services Task Force on Alzheimer's Disease. Neurology 34:939-44

14. Neary D, Snowden JS, Gustafson L et al (1998) Frontotemporal lobar degeneration: a consensus on clinical diagnostic criteria. Neurology 51:1546-54

15. van Straaten EC, Scheltens P, Knol DL et al (2003) Operational definitions for the NINDS-AIREN criteria for vascular dementia: an interobserver study. Stroke 34:1907-12

16. McKeith IG, Dickson DW, Lowe J et al (2005) Diagnosis and management of dementia with Lewy bodies: third report of the DLB Consortium. Neurology 65:1863-72

17. Freeborough PA, Fox NC (1998) Modeling brain deformations in Alzheimer disease by fluid registration of serial 3D MR images. J Comput Assist Tomogr 22:838-43
18. Fox NC, Crum WR, Scahill RI et al (2001) Imaging of onset and progression of Alzheimer's disease with voxel-compression mapping of serial magnetic resonance images. Lancet 358:201-5

19. Sled JG, Zijdenbos AP, Evans AC (1998) A nonparametric method for automatic correction of intensity nonuniformity in MRI data. IEEE Trans Med Imaging 17:87-97

20. Smith SM, Zhang Y, Jenkinson $M$ et al (2002) Accurate, robust, and automated longitudinal and cross-sectional brain change analysis. Neuroimage 17:47989

21. Lewis EB, Fox NC (2004) Correction of differential intensity inhomogeneity in longitudinal MR images. Neuroimage 23:75-83

22. Smith SM, Jenkinson M, Woolrich MW et al (2004) Advances in functional and structural MR image analysis and implementation as FSL. Neuroimage 23:S208-19

23. Tzourio-Mazoyer N, Landeau B, Papathanassiou D et al (2002) Automated anatomical labeling of activations in SPM using a macroscopic anatomical parcellation of the MNI MRI singlesubject brain. Neuroimage 15:273-89

24. Carmichael OT, Kuller LH, Lopez OL et al (2007) Ventricular volume and dementia progression in the Cardiovascular Health Study. Neurobiol Aging 28:389-397

25. van de Pol LA, Hensel A, Barkhof F et al (2006) Hippocampal atrophy in Alzheimer disease: age matters. Neurology 66:236-238

26. Apostolova LG, Steiner CA, Akopyan GG et al (2007) Three-dimensional gray matter atrophy mapping in mild cognitive impairment and mild Alzheimer disease. Arch Neurol 64:148995

27. Baron JC, Chetelat G, Desgranges B et al (2001) In vivo mapping of gray matter loss with voxel-based morphometry in mild Alzheimer's disease. Neuroimage 14:298-309

28. Karas GB, Burton EJ, Rombouts SA et al (2003) A comprehensive study of gray matter loss in patients with Alzheimer's disease using optimized voxel-based morphometry. Neuroimage 18:895-907

29. Davatzikos C, Fan Y, Wu X et al (2006) Detection of prodromal Alzheimer's disease via pattern classification of MRI. Neurobiol Aging 29:514-523

30. Whitwell JL, Shiung MM, Przybelski SA et al (2007) MRI patterns of atrophy associated with progression to $\mathrm{AD}$ in amnestic mild cognitive impairment. Neurology 70:512-520
31. Karas G, Sluimer J, Goekoop R et al (2008) Amnestic mild cognitive impairment: structural MR imaging findings predictive of conversion to Alzheimer disease. AJNR Am J Neuroradiol 29:944-949

32. Carmichael OT, Kuller LH, Lopez OL et al (2007) Cerebral ventricular changes associated with transitions between normal cognitive function, mild cognitive impairment, and dementia. Alzheimer Dis Assoc Disord 21:14-24

33. Ridha BH, Barnes J, Bartlett JW et al (2006) Tracking atrophy progression in familial Alzheimer's disease: a serial MRI study. Lancet Neurol 5:828-834

34. Scahill RI, Schott JM, Stevens JM et al (2004) Fluid registration of serial MRI: identifying regional changes in Alzheimer's disease. Neurobiol Aging 25:269-269

35. Lam LC, Lui VW, Tam CW et al (2005) Subjective memory complaints in Chinese subjects with mild cognitive impairment and early Alzheimer's disease. Int J Geriatr Psychiatry 20:87682

36. Visser PJ, Kester A, Jolles J et al (2006) Ten-year risk of dementia in subjects with mild cognitive impairment. Neurology 67:1201-7

37. Berg L, Mckeel DW Jr, Miller JP et al (1998) Clinicopathologic studies in cognitively healthy aging and Alzheimer's disease: relation of histologic markers to dementia severity, age, sex, and apolipoprotein E genotype. Arch Neurol 55:326-35

38. MRC CFAS (2001) Pathological correlates of late-onset dementia in a multicentre, community-based population in England and Wales. Neuropathology Group of the Medical Research Council Cognitive Function and Ageing Study (MRC CFAS). Lancet 357:169-75

39. Barnes J, Whitwell JL, Frost C et al (2006) Measurements of the amygdala and hippocampus in pathologically confirmed Alzheimer disease and frontotemporal lobar degeneration. Arch Neurol 63:1434-1439

40. Fearing MA, Bigler ED, Norton $M$ et al (2007) Autopsy-confirmed Alzheimer's disease versus clinically diagnosed Alzheimer's disease in the Cache County Study on Memory and Aging: a comparison of quantitative MRI and neuropsychological findings. J Clin Exp Neuropsychol 29:553-60 